\title{
Scanning Tunneling Microscopy as a Single Majorana Detector of Kitaev's Chiral Spin Liquid
}

\author{
Masafumi Udagawa, ${ }^{1,2}$ Shintaro Takayoshi, ${ }^{2,3}$ and Takashi Oka ${ }^{2,4,5}$ \\ ${ }^{1}$ Department of Physics, Gakushuin University, Mejiro, Toshima-ku, Tokyo 171-8588, Japan \\ ${ }^{2}$ Max-Planck-Institut für Physik komplexer Systeme, 01187 Dresden, Germany \\ ${ }^{3}$ Department of Physics, Konan University, Kobe, 658-8501, Japan \\ ${ }^{4}$ Institute for Solid State Physics, University of Tokyo, Kashiwa 277-8581, Japan \\ ${ }^{5}$ Trans-scale Quantum Science Institute, University of Tokyo, Bunkyo-ku, Tokyo 113-0033, Japan
}

(Received 19 August 2020; accepted 17 February 2021; published 24 March 2021)

\begin{abstract}
We propose a local detection scheme for the Majorana zero mode (MZM) carried by a vison in Kitaev's chiral spin liquid (CSL) using scanning tunneling microscopy (STM). The STM introduces a single Majorana into the system through hole-charge injection and the Majorana interacts with the MZM to form a stable composite object. We derive the exact analytical expression of single-hole Green's function in the Mott insulating limit of Kitaev's model, and show that the differential conductance has split peaks, as a consequence of resonant tunneling through the vison-hole composite. The peak splitting turns out comparable to the Majorana gap in CSL, well within the reach of experimental observation.
\end{abstract}

DOI: 10.1103/PhysRevLett.126.127201

Introduction.-In recent years, a number of candidate materials have been proposed for quantum spin liquids (QSLs), and intensive studies are going on for its realization [1]. An important class of QSL phases is characterized by their topological nature of ground states and excitations $[2,3]$. The underlying topological structure means the robustness of the phase, as local perturbations cannot change the global topology immediately. However, due to its inherent nonlocality, the topological structure sometimes disables direct access by local experimental probes and makes it a challenging task to identify the QSL phase experimentally.

Among many QSL candidates proposed so far, Kitaev's chiral spin liquid (CSL) state deserves special attention [4]. The appearance of the CSL phase is theoretically predicted for Kitaev's honeycomb model in a magnetic field. This phase hosts a finite Chern number, which results in halfinteger quantization of thermal Hall conductivity, as recently claimed in the field-induced nonmagnetic state [5,6] of $\alpha-\mathrm{RuCl}_{3}$ [7-9]. Moreover, due to the finite Chern number of the phase, the $Z_{2}$ vortex excitation called a vison, is turned into a non-Abelian Ising anyon accompanied with a Majorana zero mode (MZM).

Published by the American Physical Society under the terms of the Creative Commons Attribution 4.0 International license. Further distribution of this work must maintain attribution to the author(s) and the published article's title, journal citation, and DOI. Open access publication funded by the Max Planck Society.
Detection and control of MZM opens an avenue to perform a topological quantum computation, and its impact on material physics and quantum information technology is considerable [10-12]. Indeed, great efforts have been made for the identification of MZM through local spectroscopic measurements [13-22], such as scanning tunneling microscopy (STM). Target systems range from quantum wires [10,13-18], vortices in topological superconductors [18-32], surface states of ${ }^{3} \mathrm{He}$ and topological insulators [33-36], to non-Abelian fractional Hall states [37-39]. In superconductors, MZM is a superposition of electrons and holes, and STM can access it directly. However, in the CSL phase, no local observables couple with a spatially isolated MZM, which is only a half of physical spin degrees of freedom. Hence MZM in the CSL has been elusive so far. In this work, we shed a new light on this problem through the hole-charge injection to the system. Recently, mobile carrier doping has been attempted for $\alpha-\mathrm{RuCl}_{3}$ [40-44]. Theoretically, the possibility of binding a vison to a static hole (vacancy) has been discussed in the anisotropic $A$ phase and gapless $B$ phase of Kitaev's spin liquid and related models [45-50]. However, the interaction between an injected hole-charge and vison remains largely unexplored in the CSL.

In this Letter, we propose a detection scheme of MZM using STM. In the CSL phase, MZM is known to accompany a vison [51], or a spin vacancy $[45,46]$. Our idea is to utilize the MZM of the latter to detect the MZM of the former. Tunneling current of STM introduces a spin vacancy as an injected hole. Then, one can detect MZM by observing a bonding state between a MZM carried by the hole and the target MZM attached to a vison. This bonding 


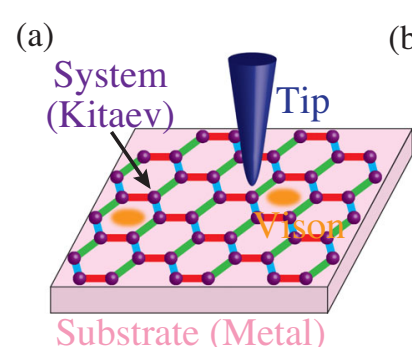

(b)

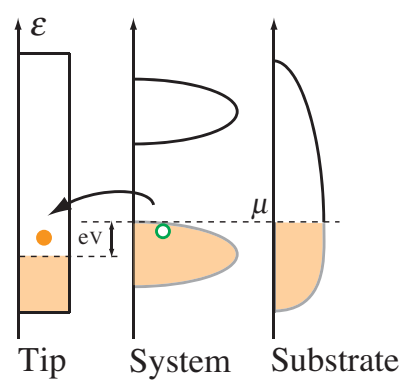

(c)

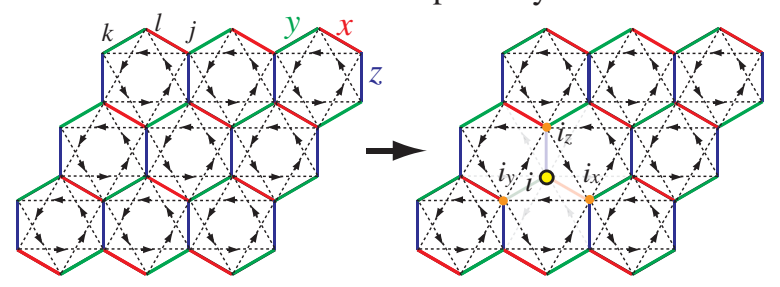

FIG. 1. (a) Theoretical setup of STM device. Electric current conducts from an STM tip to a metallic substrate through the Kitaev's system. (b) Schematic plot of DOS and electron occupation. $\mu$ and $V$ means the chemical potential and the voltage bias (see main text). (c) Lattice convention of Kitaev's Hamiltonian. Red, green, and blue bonds represent the Ising couplings of $x, y$, and $z$ components. The dashed lines express the hopping direction of Majoranas, due to the pseudomagnetic field terms $(\propto \kappa)$. To introduce a hole at site $i$, we remove the bonds to its neighbors, $i_{x}, i_{y}$, and $i_{z}$.

state is more robust than the vison-vacancy composites in $A$ and $B$ phases; the binding energy is comparable to the Majorana gap of CSL, i.e., the composite is well stabilized, as soon as the CSL phase is realized with a well-defined excitation gap.

Model.-As a theoretical model of STM, we consider the setup shown in Fig. 1(a): we place a target sample to realize Kitaev CSL on a metallic substrate, and allow conduction of the electric current from the STM tip to the substrate through the sample. We model this device by the Hamiltonian,

$$
\mathcal{H}=\mathcal{H}_{\text {sys }}+\mathcal{H}_{\text {tip }}+\mathcal{H}_{\text {sub }}+\mathcal{H}_{\text {hyb }}^{\text {tip }}+\mathcal{H}_{\text {hyb }}^{\text {sub }} .
$$

Here, $\mathcal{H}_{\text {sys }}$ represents the Kitaev's system,

$$
\mathcal{H}_{\text {sys }}=\mathcal{P} \mathcal{H}_{K} \mathcal{P}+\mathcal{P} \mathcal{H}_{\text {hop }} \mathcal{P} .
$$

$\mathcal{H}_{K}$ is the Kitaev's effective Hamiltonian with the pseudomagnetic field term to realize the CSL phase,

$$
\mathcal{H}_{K}=-J_{K} \sum_{\langle i, j\rangle_{\alpha}} S_{j}^{\alpha} S_{k}^{\alpha}-2 \kappa \sum_{\langle j, k, l\rangle_{\alpha \beta}} S_{j}^{\alpha} S_{k}^{\beta} S_{l}^{\gamma},
$$

where $S_{j}^{\alpha}$ is the spin-1/2 operator in fermionic representation, $S_{j}^{\alpha} \equiv \frac{1}{2} f_{j, s}^{\dagger} \sigma_{s s^{\prime}}^{\alpha} f_{j, s^{\prime}}$, with $f_{j, s}^{\dagger}$, the creation operator of a fermion at site $j$ and spin $s$. We focus on the case of hole injection into the half-filled Mott insulating state; $\mathcal{P}$ is the projection operator to exclude doublons. $\mathcal{H}_{\text {hop }}$ describes the motion of an injected hole.
$\mathcal{H}_{\text {tip }}=\sum_{m \sigma} E_{m}^{t} \alpha_{m \sigma}^{\dagger} \alpha_{m \sigma}$ and $\mathcal{H}_{\text {sub }}=\sum_{l \sigma} E_{l}^{s} \beta_{l \sigma}^{\dagger} \beta_{l \sigma}$ are the Hamiltonians of the tip and the substrate, modeled as simple noninteracting metals, characterized by the density of states (DOS), $\rho^{\mathrm{t}}(\varepsilon)$ and $\rho^{\mathrm{s}}(\varepsilon)$, respectively. $\mathcal{H}_{\text {hyb }}^{\mathrm{tip}}=\sum_{m \sigma} v_{m \sigma}^{\mathrm{t}}\left(\alpha_{m \sigma}^{\dagger} f_{i \sigma}+\right.$ H.c. $)$ describes the tunneling of electrons between the tip and Kitaev's system through the site $i$ in the Kitaev's system, and $\mathcal{H}_{\mathrm{hyb}}^{\text {sub }}$ accounts for the tunneling process between the substrate and the system.

The tunneling current of STM can be written as [52-55]

$$
I_{i}=-\frac{2 \pi e}{\hbar}|v|^{2} \int d \omega \rho^{\mathrm{t}}(\omega+e V) \rho_{i}(\omega)[f(\omega+e V)-f(\omega)],
$$

where $\rho_{i}(\omega)$ is the local DOS of Kitaev's system at site $i$, in contact with the tip [56]. The tunneling amplitude is set to be constant: $v_{m \sigma}^{\mathrm{t}}=v$, for simplicity. We assume the Kitaev's system is in equilibrium with the substrate, and their common chemical potential $\mu$ is tuned at the lower edge of the charge gap, which may originate from a MottHubbard splitting. Meanwhile, we set a voltage bias $V$ between the tip and the system [Fig. 1(b)], which drives the electric current between the system and tip, through the difference of distribution functions of the system, $f(\varepsilon)=1 /\left[e^{\beta(\varepsilon-\mu)}+1\right]$ and of the tip, $f(\varepsilon+e V)=$ $1 /\left[e^{\beta(\varepsilon+e V-\mu)}+1\right]$, respectively.

By assuming constant DOS: $\rho^{\mathrm{t}}(\varepsilon)=\bar{\rho}$, the electric current can be simplified as $I_{i}=-(2 \pi e / \hbar)|v|^{2} \bar{\rho} \int_{\mu}^{\mu-e V} \rho_{i}(\varepsilon) d \varepsilon$ at zero temperature. Accordingly, the differential conductance $d I / d V$ gives direct information on the local DOS, $\rho_{i}(\mu-e V)$.

We introduce the hole Green's function, $g_{i \sigma}(t) \equiv$ $-i\left\langle\Omega\left|f_{i \sigma}^{\dagger}(t) f_{i \sigma}(0)\right| \Omega\right\rangle$, which gives local DOS through $\rho_{i}(\varepsilon)=-(1 / \pi) \sum_{\sigma} \operatorname{Im} g_{i \sigma}(\varepsilon)$. Here, $|\Omega\rangle$ represents the ground state of $\mathcal{H}_{\text {sys }}$ at half filling (one fermion per site), which is nothing but the ground state of Kitaev's Hamiltonian, $\mathcal{H}_{K}$.

To obtain $g_{i \sigma}(t)$, we need to diagonalize the Hamiltonian, $\mathcal{H}_{\text {sys }}$ [Eq. (2)], in which $\mathcal{H}_{\text {hop }}$ represents the motion of an injected hole in the system. Below, we ignore $\mathcal{H}_{\text {hop }}$, by assuming the influence of hole motion on the spin state is small, compared with magnetic interaction. Under this assumption, the injected hole can be regarded as a site vacancy, and we can describe the intermediate state, $f_{i \sigma}(0)|\Omega\rangle$, as a superposition of the eigenstates of Kitaev's Hamiltonian, $\mathcal{H}_{K}$, in the presence of a vacancy at site $i$. Without $\mathcal{H}_{\text {hop }}$, doublons are automatically removed.

To diagonalize $\mathcal{H}_{K}$, we adopt Kitaev's Majorana representation of spin- $1 / 2$ operator, $S_{j}^{\alpha}=(i / 2) c_{j} b_{j}^{\alpha}$, and rewrite the Hamiltonian $\mathcal{H}_{K}$ as

$$
\mathcal{H}_{K}=\frac{i}{4} \sum_{j j^{\prime}} c_{j} A_{j j^{\prime}} c_{j^{\prime}}=\sum_{m: \varepsilon_{m}>0} \varepsilon_{m}\left(\gamma_{m}^{\dagger} \gamma_{m}-\frac{1}{2}\right)
$$


where we have introduced complex fermion operators, $\gamma_{m}$. The site vacancy modifies the Hamiltonian matrix, $A$. Here, the first and second terms of $\mathcal{H}_{K}$ in Eq. (3) are transformed into nearest- and next-nearest-neighbor hoppings of $c$ Majorana, $c_{j}$, as schematically shown in Fig. 1(c). The $b$ Majoranas, $b_{j}^{\alpha}$, combine into gauge fluxes defined on each hexagon and appears as a phase in $A$. The $\pi$-vortex of gauge fluxes, called vison, increases the system energy by affecting the Hamiltonian matrix of $c$-Majorana, $A[59,60]$. An isolated vison accompanies one MZM in the CSL phase $(\kappa \neq 0)$, in the same mechanism as chiral $p$-wave superconductors [23] and Moore-Read fractional quantum Hall state [37].

One of the authors recently developed a technique to obtain a dynamical correlation function on a real-time basis $[49,61]$. Using the technique, the hole Green's function is expressed as [56]

$$
g_{i \sigma}(t)=-\frac{i}{2} \frac{\sqrt{\operatorname{det}\left(1+e^{-(\beta-i t) \cdot i A} e^{-i t \cdot i A^{i}}\right)}+(-1)^{F} \sqrt{\operatorname{det}\left(1-e^{-(\beta-i t) \cdot i A} e^{-i t \cdot i A^{i}}\right)}}{\sqrt{\operatorname{det}\left(1+e^{-\beta \cdot i A}\right)}+(-1)^{F} \sqrt{\operatorname{det}\left(1-e^{-\beta \cdot i A}\right)}},
$$

where $(-1)^{F}$ is the physical fermion parity $[49,62] . A^{i}$ and $A$ are the Hamiltonian matrices in Eq. (4) with and without a static hole at site $i$. We consider two cases: (i) the flux free case; no hexagonal plaquette supports a vison, and (ii) the isolated vison case; a vison is introduced at the target site with its counterpart of the pair placed with maximal separation. Difference of two cases are reflected in $A^{i}$ and $A$, i.e., we consider four types of matrices, depending on the presence or absence of hole and vison. See Ref. [56].

Hereafter, we consider the system of $N \times N$ unit cells in the periodic boundary condition, with $N=48$. We set $J_{K}=1$ as a unit of energy. For the computation of $g_{i \sigma}(t)$ in Eq. (5), we set the inverse temperature, $\beta=50$, where only a small number of visons are thermally excited.

Results.-Figure 2 represents our central result. Here, we set the tip at site $i=0$, and compare the local DOS, $\rho_{0}(\varepsilon)$ for the flux free case and the isolated vison case, for $\kappa=0.1$. In the latter case, we inject a hole on a site shared by the vison [Fig. 2, inset]. The two spectra show a clear difference. While the flux-free spectrum shows a single broad peak at $\varepsilon=\varepsilon_{\mathrm{ff}} \sim 0.37$, the spectrum splits into two peaks in the presence of a vison. This clear difference

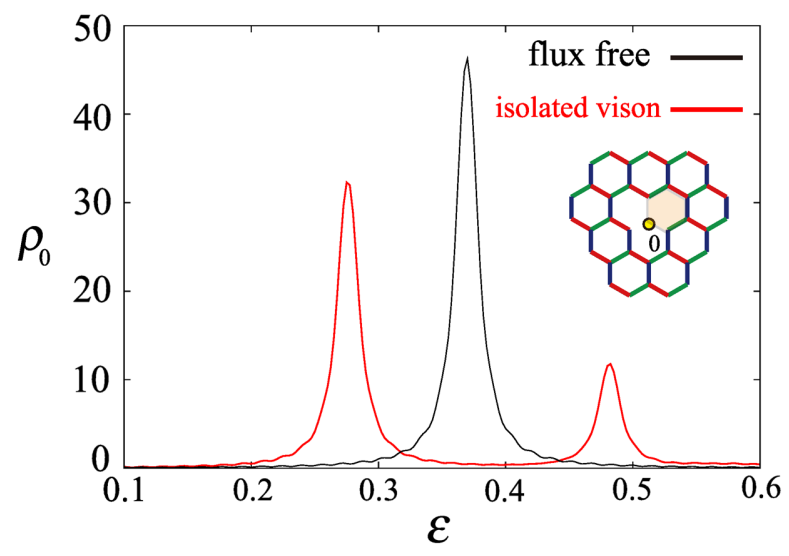

FIG. 2. Local density of state, $\rho_{0}(\varepsilon)$ for $\kappa=0.1$ at the neighboring site of a single vison (red), and in the flux free case (black). The inset shows the target site $i=0$ with a yellow circle, and a vison with red shade. can be accessed through the differential conductance, $d I / d V \propto \rho_{0}(\mu-e V)$, and thus it provides a clear diagnosis of the presence of a vison.

To understand this spectral feature, we start with the flux free case. The physical origin of the single peak can be understood as follows. Since we consider the limit where the charge motion is frozen, the spectrum is dominated by the magnetic excitation caused by the hole injection. The localized spin is removed by the hole injection, and this effectively disconnects three bonds. Indeed, the resonant energy of the flux-free spectrum is well estimated as $\varepsilon_{\mathrm{ff}} \sim$ $J_{K} \sum_{\alpha=x, y, z}\left\langle s_{i}^{\alpha} s_{i_{\alpha}}^{\alpha}\right\rangle \sim 0.39$ from the first-order perturbation by the removed bonds [Fig. 1(c)].

Then, how does the presence of vison split this peak? To see its origin, we plot $\rho_{0}(\varepsilon)$ in the presence of the vison as changing the pseudomagnetic field term $\kappa$ in Fig. 3(a). For $\kappa=0$, the spectrum consists of only a single peak at $\varepsilon \sim 0.3$, as in the flux free case. However, for finite $\kappa$, it splits into one low-energy main peak and one high-energy subdominant peak, and the separation between the peaks becomes larger as increasing $\kappa$.

The low-energy main peak barely shifts from the original single peak position at $\kappa=0$, even when $\kappa$ is increased. As explained above, the main peak originates from the release of the spin correlation energy. Meanwhile, the subdominant peak quickly shifts to higher energy, when we increase $\kappa$.

This subdominant peak reflects the information of the MZM carried by the vison. The key to understanding this is that the injected hole creates additional MZMs in the system, and interacts with the MZM attached to a vison, if it exists near the tip. To clarify this, let us compare the energy level structures of the complex fermions $\left(\gamma_{m}\right)$ in the absence and presence of the vison. In Fig. 3(b), we show the energy levels after introducing a hole in the flux free state. A finite excitation gap $\Delta=(3 \sqrt{3} / 4) \kappa$, known as the Majorana gap, opens in the spectrum in the absence of the hole. When the hole is introduced, there appears a MZM inside the gap as depicted with a red line in Fig. 3(b). It is an unpaired Majorana left in the bulk: Removal of one Majorana leaves an odd number of Majorana modes locally 

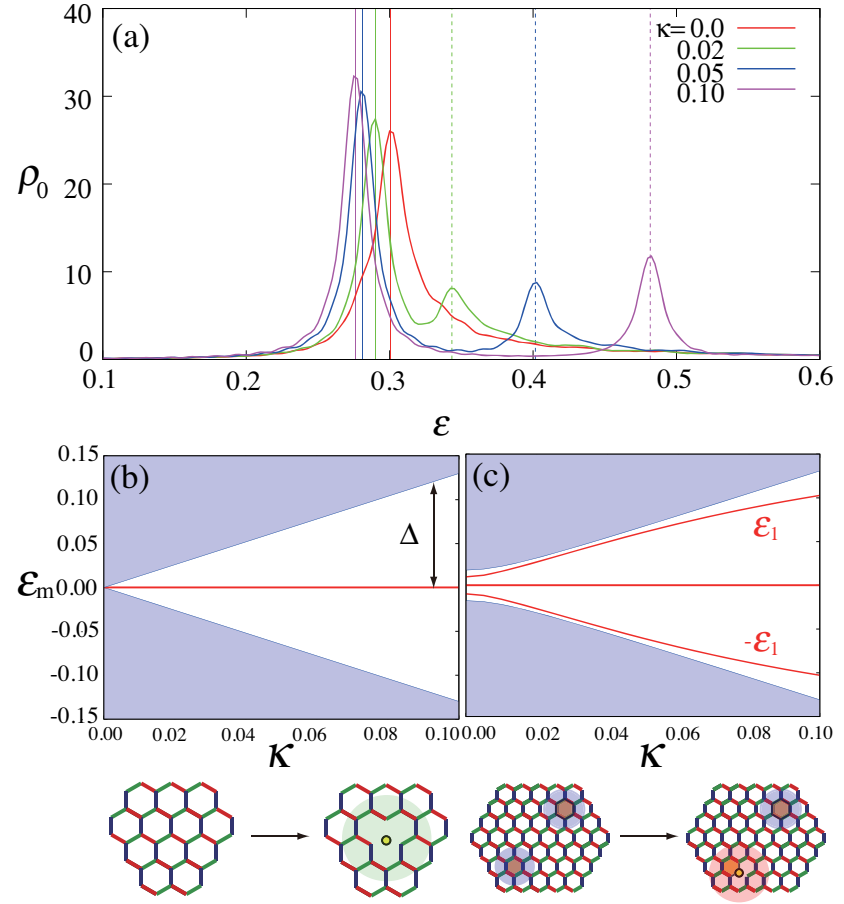

FIG. 3. (a) Local DOS at the neighboring site of single vison for $\kappa=0.0,0.02,0.05$. and 0.10 . The vertical lines are obtained from Eq. (6) with (solid line) $\delta \varepsilon^{(f)}=0$ and (dashed line) $2 \varepsilon_{1}$, respectively. (b) and (c) The numerically obtained energy levels after the hole injection (b) in the flux free, and (c) in the isolated vison case. The high-energy continuum is shown with blue shading. The low-energy modes are shown with red lines. (b) One mode appears at $\varepsilon=0$. (c) There exists a mode at $\varepsilon=0$, and the antibonding-bonding states at $\pm \varepsilon_{1}$. The schematic figures of visons and a hole are shown before and after the introduction of a hole.

around the vacancy site, leading inevitably to a formation of one zero mode. It means the injection of a hole practically introduces a single unpaired Majorana in the bulk.

This unpaired Majorana leads to a nontrivial result in the presence of visons [Fig. 3(c)]. To see this, let us assume that there are a pair of visons. If two visons are well separated, there exist a pair of MZMs, each attached to the visons, and the lift of their energies from zero is exponentially small determined by their separation. Next, let us consider adding a hole near one of the visons. As we explained above, the hole adds one unpaired MZM, which then accompanies the MZM of the vison. The two MZMs form a bonding and antibonding pair whose energies are denoted as $\pm \varepsilon_{1}$ [Fig. 3(c)]. The formation of this bonding-antibonding state opens a new channel of fermion excitations, which brings about the high-energy second peak in $\rho_{0}(\varepsilon)$.

To verify this scenario, let us look at the one-particle spectrum of Majorana Hamiltonian, Eq. (4). In this representation, the resonant energy can be written as

$$
\omega_{\text {res }}^{i \rightarrow f}=\left(E_{\mathrm{ZP}}^{(f)}-E_{\mathrm{ZP}}^{(i)}\right)+\delta \varepsilon^{(f)},
$$

where the first term is concerned with the change of vacuum energy, $E_{\mathrm{ZP}} \equiv-\frac{1}{2} \sum_{m: \varepsilon_{m}>0} \varepsilon_{m}$, obtained from Eq. (4). The introduction of a hole affects all the energy levels, resulting in the modification of $E_{\mathrm{ZP}}$. The second term, $\delta \varepsilon^{(f)}$, accounts for the changes in occupation numbers of fermions at each energy level.

On the basis of Eq. (6), we can attribute the low-energy main peaks to the vacuum-to-vacuum transition. We obtain $\Delta E_{0} \equiv E_{\mathrm{ZP}}^{(f)}-E_{\mathrm{ZP}}^{(i)}$ for each $\kappa$, and plot them by vertical solid lines in Fig. 3(a), which match the position of resonant peaks accurately.

In contrast, the high-energy second peaks involve fermion excitation. As mentioned above, at finite $\kappa$, the bonding between the injected unpaired MZM and the vison MZM results in discrete levels at $\pm \varepsilon_{1}$. Accordingly, we can again reproduce the position of second peaks in Fig. 3(a) with the transition involving the fermionic excitation in the antibonding level, the vertical dashed lines, corresponding to $\Delta E_{2} \equiv\left(E_{\mathrm{ZP}}^{(f)}-E_{\mathrm{ZP}}^{(i)}\right)+2 \varepsilon_{1}$.

These two successful comparisons mean the separation between two resonant peaks scales with the bonding energy, $2 \varepsilon_{1}$. As shown in Fig. 3(c), $\varepsilon_{1}$ is of the order of Majorana gap $\Delta$ proportional to $\kappa . \kappa$ is usually considered to be small, proportional to the cubic of a magnetic field, $h^{3}$ [4], however, off-diagonal interactions may enhance its magnitude [63,64], and in fact, the estimate of $\Delta \sim 10 \mathrm{~K}$ is proposed from the recent field-angle dependent specific heat [65]. This large bonding energy of the CSL phase is in sharp contrast to the energy scale of vison-vacancy composites discussed in the $\mathrm{A}$ and $B$ phases, where the perturbative arguments result in $\sim 10^{-6} J_{z}$ for the $A$ phase and $0.027 J_{K}[45,46]$ for the $B$ phase, respectively. Compared with these cases, the stability of the vison-hole composite in the CSL phase is much more robust, promising clearer experimental identification of MZM.

What happens if the STM tip is near but away from the vison? To clarify this, we inject the hole on sites $i$ away from the vison and plot the spectra $\rho_{i}(\varepsilon)$ in Fig. 4. As we move away from the hexagon supporting the vison, the two peaks quickly merge and the spectrum converges to the one in the flux-free case [56]. As a hole goes away from the vison, the coupling between MZMs becomes weaker, resulting in smaller bonding energy. The length scale of peak collapse depends on the excitation gap. This sensitive spatial dependence of $\rho_{i}(\varepsilon)$, relative to the location of vison, can be detected by sweeping the STM tip across the sample and provide useful information on the position of visons.

Summary.-We have considered the hole injection into the Kitaev's CSL phase, in the experimental setting of STM. We found that an injected hole effectively introduces a single Majorana into the system, and it forms a stable composite object with a precedent Majorana zero mode attached to a vison. The bonding energy of two Majoranas is of the order of Majorana gap of the CSL phase and is 


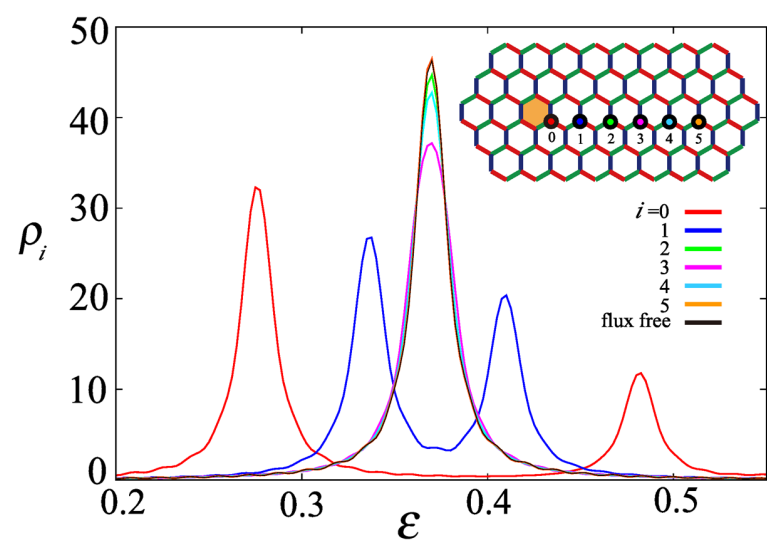

FIG. 4. Spatial dependence of local DOS, $\rho_{i}(\varepsilon)$. Site index convention is shown in the inset, together with the position of vison. Away from the vison, $\rho_{i}(\varepsilon)$ approaches the form of fluxfree case, represented by a black line.

much larger than the binding energy of vison to a vacancy in the $A$ and $B$ phases. The composite results in a doublepeak structure of the local DOS, which can be observed through the differential conductance. The local DOS, with its characteristic magnetic field and spatial dependence, gives a good diagnosis of the presence of MZM attached to a vison. In a broader context, our experimental scheme may be extended to a broad range of QSL systems, where the excitation spectrum similarly consists of matter fields accompanied with gauge fluxes.

The use of STM has been recently proposed for the detection of edge states and fractional excitations [66,67]. In a broader scope, the local charge response of the magnet may provide an access to "nonlocal" information in terms of the magnetic degrees of freedom. The recent rapid development of local charge sensitive probes may open an avenue to the measurement of nonlocal topological order, and to the long-awaited experimental identification of quantum spin liquids and their fascinating elementary excitations.

We deeply acknowledge Y. Matsuda, M. Knap, K. Damle, P. Wahl, P. A. McClarty, and J. Knolle for helpful discussions. This work was supported by JSPS KAKENHI (No. JP15H05852 and No. JP16H04026), MEXT, Japan, and JST CREST Grant No. JPMJCR19T3, Japan.

[1] L. Balents, Nature (London) 464, 199 (2010).

[2] X.-G. Wen, Quantum Field Theory of Many-Body Systems: From the Origin of Sound to an Origin of Light and Electrons (Oxford University Press on Demand, Oxford, 2004).

[3] J. K. Pachos, Introduction to Topological Quantum Computation (Cambridge University Press, Cambridge, England, 2012).

[4] A. Kitaev, Ann. Phys. (Amsterdam) 321, 2 (2006).
[5] A. Banerjee, P. Lampen-Kelley, J. Knolle, C. Balz, A. A. Aczel, B. Winn, Y. Liu, D. Pajerowski, J. Yan, C. A. Bridges, A. T. Savici, B. C. Chakoumakos, M. D. Lumsden, D. A. Tennant, R. Moessner, D. G. Mandrus, and S. E. Nagler, npj Quantum Mater. 3, 8 (2018).

[6] M. Majumder, M. Schmidt, H. Rosner, A. A. Tsirlin, H. Yasuoka, and M. Baenitz, Phys. Rev. B 91, 180401(R) (2015).

[7] Y. Kasahara, T. Ohnishi, Y. Mizukami, O. Tanaka, S. Ma, K. Sugii, N. Kurita, H. Tanaka, J. Nasu, Y. Motome, T. Shibauchi, and Y. Matsuda, Nature (London) 559, 227 (2018).

[8] M. Yamashita, N. Kurita, and H. Tanaka, Phys. Rev. B 102, 220404 (2020).

[9] T. Yokoi, S. Ma, Y. Kasahara, S. Kasahara, T. Shibauchi, N. Kurita, H. Tanaka, J. Nasu, Y. Motome, C. Hickey, S. Trebst, and Y. Matsuda, arXiv:2001.01899.

[10] A. Y. Kitaev, Phys. Usp. 44, 131 (2001).

[11] A. Kitaev, Ann. Phys. (Amsterdam) 303, 2 (2003).

[12] C. Nayak, S. H. Simon, A. Stern, M. Freedman, and S. Das Sarma, Rev. Mod. Phys. 80, 1083 (2008).

[13] V. Mourik, K. Zuo, S. M. Frolov, S. R. Plissard, E. P. A. M. Bakkers, and L. P. Kouwenhoven, Science 336, 1003 (2012).

[14] S. Nadj-Perge, I. K. Drozdov, J. Li, H. Chen, S. Jeon, J. Seo, A. H. MacDonald, B. A. Bernevig, and A. Yazdani, Science 346, 602 (2014).

[15] S. M. Albrecht, A. P. Higginbotham, M. Madsen, F. Kuemmeth, T. S. Jespersen, J. Nygård, P. Krogstrup, and C. M. Marcus, Nature (London) 531, 206 (2016).

[16] H. Kim, A. Palacio-Morales, T. Posske, L. Rózsa, K. Palotás, L. Szunyogh, M. Thorwart, and R. Wiesendanger, Sci. Adv. 4, eaar5251 (2018).

[17] D. Chevallier and J. Klinovaja, Phys. Rev. B 94, 035417 (2016).

[18] J. J. He, T. K. Ng, P. A. Lee, and K. T. Law, Phys. Rev. Lett. 112, 037001 (2014).

[19] T. Machida, Y. Sun, S. Pyon, S. Takeda, Y. Kohsaka, T. Hanaguri, T. Sasagawa, and T. Tamegai, Nat. Mater. 18, 811 (2019).

[20] D. Wang, L. Kong, P. Fan, H. Chen, S. Zhu, W. Liu, L. Cao, Y. Sun, S. Du, J. Schneeloch, R. Zhong, G. Gu, L. Fu, H. Ding, and H.-J. Gao, Science 362, 333 (2018).

[21] M. Chen, X. Chen, H. Yang, Z. Du, X. Zhu, E. Wang, and H.-H. Wen, Nat. Commun. 9, 970 (2018).

[22] H.-H. Sun, K.-W. Zhang, L.-H. Hu, C. Li, G.-Y. Wang, H.-Y. Ma, Z.-A. Xu, C.-L. Gao, D.-D. Guan, Y.-Y. Li, C. Liu, D. Qian, Y. Zhou, L. Fu, S.-C. Li, F.-C. Zhang, and J.-F. Jia, Phys. Rev. Lett. 116, 257003 (2016).

[23] D. A. Ivanov, Phys. Rev. Lett. 86, 268 (2001).

[24] M. Sato and S. Fujimoto, Phys. Rev. B 79, 094504 (2009).

[25] M. Sato, Y. Takahashi, and S. Fujimoto, Phys. Rev. B 82, 134521 (2010).

[26] M. Sato and S. Fujimoto, Phys. Rev. Lett. 105, 217001 (2010).

[27] J. D. Sau, R. M. Lutchyn, S. Tewari, and S. Das Sarma, Phys. Rev. Lett. 104, 040502 (2010).

[28] R. M. Lutchyn, J. D. Sau, and S. Das Sarma, Phys. Rev. Lett. 105, 077001 (2010).

[29] M. Cheng, R. M. Lutchyn, V. Galitski, and S. Das Sarma, Phys. Rev. B 82, 094504 (2010).

[30] T. Liu and M. Franz, Phys. Rev. B 92, 134519 (2015).

[31] R. R. Biswas, Phys. Rev. Lett. 111, 136401 (2013). 
[32] T. Yoshida and M. Udagawa, Phys. Rev. B 94, 060507(R) (2016).

[33] S. Murakawa, Y. Wada, Y. Tamura, M. Wasai, M. Saitoh, Y. Aoki, R. Nomura, Y. Okuda, Y. Nagato, M. Yamamoto, S. Higashitani, and K. Nagai, J. Phys. Soc. Jpn. 80, 013602 (2011).

[34] H. Ikegami and K. Kono, J. Low Temp. Phys. 195, 343 (2019).

[35] Y. Tsutsumi, Phys. Rev. Lett. 118, 145301 (2017).

[36] L. Fu and C. L. Kane, Phys. Rev. Lett. 100, 096407 (2008).

[37] G. Moore and N. Read, Nucl. Phys. B360, 362 (1991).

[38] P. Bonderson, A. Kitaev, and K. Shtengel, Phys. Rev. Lett. 96, 016803 (2006).

[39] R. L. Willett, L. N. Pfeiffer, and K. W. West, Proc. Natl. Acad. Sci. U.S.A. 106, 8853 (2009).

[40] A. Koitzsch, C. Habenicht, E. Müller, M. Knupfer, B. Büchner, S. Kretschmer, M. Richter, J. van den Brink, F. Börrnert, D. Nowak, A. Isaeva, and T. Doert, Phys. Rev. Mater. 1, 052001(R) (2017).

[41] Y. Imai, K. Konno, Y. Hasegawa, T. Aoyama, and K. Ohgushi, Phys. Rev. B 99, 245141 (2019).

[42] L. Wang, M. Rocci-Lane, P. Brazis, C. R. Kannewurf, Y.-I. Kim, W. Lee, J.-H. Choy, and M. G. Kanatzidis, J. Am. Chem. Soc. 122, 6629 (2000).

[43] B. Zhou, J. Balgley, P. Lampen-Kelley, J.-Q. Yan, D. G. Mandrus, and E. A. Henriksen, Phys. Rev. B 100, 165426 (2019).

[44] S. Mashhadi, Y. Kim, J. Kim, D. Weber, T. Taniguchi, K. Watanabe, N. Park, B. Lotsch, J. H. Smet, M. Burghard, and K. Kern, Nano Lett. 19, 4659 (2019).

[45] A. J. Willans, J. T. Chalker, and R. Moessner, Phys. Rev. Lett. 104, 237203 (2010).

[46] A. J. Willans, J. T. Chalker, and R. Moessner, Phys. Rev. B 84, 115146 (2011).

[47] G. B. Halász, J. T. Chalker, and R. Moessner, Phys. Rev. B 90, 035145 (2014).

[48] G. B. Halász and J. T. Chalker, Phys. Rev. B 94, 235105 (2016).
[49] M. Udagawa, Phys. Rev. B 98, 220404(R) (2018).

[50] S. Sanyal, K. Damle, J. Chalker, and R. Moessner, arXiv:2006.16987.

[51] See the Appendix C of Ref. [4].

[52] J. Bardeen, Phys. Rev. Lett. 6, 57 (1961).

[53] J. Tersoff and D. R. Hamann, Phys. Rev. B 31, 805 (1985).

[54] N. D. Lang, Phys. Rev. B 34, 5947 (1986).

[55] M. Maltseva, M. Dzero, and P. Coleman, Phys. Rev. Lett. 103, 206402 (2009).

[56] Supplemental Material at http://link.aps.org/supplemental/10 .1103/PhysRevLett.126.127201 (which also cites Refs. [57,58]) for derivation of STM current and hole Green's function, configuration of visons, example of Hamiltonian matrices and spatial profile of Majorana wavefunctions.

[57] A. Kamenev, Field Theory of Non-Equilibrium Systems (Cambridge University Press, Cambridge, England, 2011).

[58] V. M. Pereira, F. Guinea, J. M. B. Lopes Dos Santos, N. M. R. Peres, and A. H. Castro Neto, Phys. Rev. Lett. 96, 036801 (2006).

[59] V. Lahtinen, G. Kells, A. Carollo, T. Stitt, J. Vala, and J. K. Pachos, Ann. Phys. (Amsterdam) 323, 2286 (2008).

[60] V. Lahtinen, New J. Phys. 13, 075009 (2011).

[61] M. Udagawa and R. Moessner, arXiv:1912.01545.

[62] F. L. Pedrocchi, S. Chesi, and D. Loss, Phys. Rev. B 84, 165414 (2011).

[63] D. Takikawa and S. Fujimoto, Phys. Rev. B 99, 224409 (2019).

[64] D. Takikawa and S. Fujimoto, Phys. Rev. B 102, 174414 (2020).

[65] O. Tanaka, Y. Mizukami, R. Harasawa, K. Hashimoto, N. Kurita, H. Tanaka, S. Fujimoto, Y. Matsuda, E. G. Moon, and T. Shibauchi, arXiv:2007.06757.

[66] M. Carrega, I. J. Vera-Marun, and A. Principi, Phys. Rev. B 102, 085412 (2020).

[67] J. Feldmeier, W. Natori, M. Knap, and J. Knolle, Phys. Rev. B 102, 134423 (2020). 